\title{
FUNCTIONAL REPRESENTATION OF VECTOR LATTICES
}

\author{
ISIDORE FLEISCHER
}

(Communicated by John B. Conway)

\begin{abstract}
Every vector lattice is represented in the lattice of distribution functions valued in the complete Boolean algebra of its annihilators; the representation is complete join and positive multiple preserving and subadditive; restricted to the solid vector sublattice without infinitesimals, it preserves the full structure (including any existing infinite lattice extrema) and is faithful. Identifying the distribution with the continuous real-valued functions on the extremally disconnected Stone space of the algebra yields a representation which, specialized to Archimedean vector lattices, embeds them in the densely finite-valued continuous functions; identifying with the equivalence classes of functions measurable for a $\sigma$-field modulo a $\sigma$-ideal of "null sets" yields a representation which, specialized to Archimedean vector lattices, embeds them in the classes of a.e. finite functions. This is used to give simple proofs of Freudenthal's spectral theorem and Kakutani's structure theorem for $L$-spaces.
\end{abstract}

\section{THE LATTICE-ORDERED MONOID OF DISTRIBUTION FUNCTIONS}

An extended real-valued function on a set $X$ is determined by the subsets $[f \prec \lambda]$ of elements at which $f$ is less than a rational $\lambda$-or alternatively those at which $f$ is less than or equal to $\lambda$. These forms are isotone in $\lambda$ and antitone in $f$. Abstracting, one could construe any isotone map from the rationals into a poset as (a representation of) such a (real-valued) "function". The resulting system of "distribution" or "place" functions is preordered by $f \leq g$ when $[g \prec \mu] \leq[f \prec \lambda]$ for every pair $\mu<\lambda$; one will want to construe the actual functions as their equivalence classes under $g \leq f$ and $f \leq g$. This makes their totality (for a fixed poset) partially ordered; they admit positive real scalar multiplication; and if this poset is a lattice, they are lattice-ordered by virtue of $[f \vee g \prec \lambda]=[f \prec \lambda] \wedge[g \prec \lambda],[f \wedge g \prec \lambda]=[f \prec \lambda] \vee[g \prec \lambda]$. In a $\sigma$-lattice they form a $\sigma$-lattice; moreover one then has for single functions $\bigvee[f \prec \mu]: \mu<\lambda \leq[f \prec \lambda] \leq \Lambda[f \prec \mu]: \mu>\lambda$. One could arrange to have either of these an equality by passing to an equivalent representation for the function - the $\Lambda$ would give a true interpretation of $\{f \leq \lambda\}$, the $\vee$ of

Received by the editors April 28, 1989.

1980 Mathematics Subject Classification (1985 Revision). Primary 46A40, 06F20; Secondary 46B30, 46E05, 46E30, 54C30, 54H10. 
$\{f<\lambda\}$ (even for nonrational, real $\lambda$ ). It is now also possible to define addition of functions as $[f+g \prec \nu]=\bigvee[f \prec \lambda] \wedge[g \prec \mu]: \lambda+\mu<\nu .{ }^{1}$ This $\bigvee$ has a countable cofinal set of terms, hence exists in a $\sigma$-lattice; it is isotone in $\nu$, hence represents a function. Addition is clearly commutative and orderpreserving; when the $\sigma$-lattice is $\sigma$-continuous, i.e. meet distributes across sups of increasing sequences, also associative. Requiring next that the lattice have a least element $\varnothing$, one can define when a function is positive $-[f \prec \lambda]=\varnothing$ for $\lambda<0$-and has the function constant $k$ on an element $E$ of the lattice, as that for which $[f \prec \lambda]=\varnothing$ for $\lambda<k$ and $E$ for $\lambda>k$; when there is also a greatest element $X$ one gets also the constant function 0 , which is an additive identity. Finally, if the lattice admits complements for the $[f \prec \lambda]$, one could define $[-f \prec \lambda]$ as $[f \prec-\lambda]^{\perp}$. It is readily verified that negation is an order-inverting involution; moreover it subdistributes across sum: $-(f+g) \leq-f+-g$. Indeed, $\left.[-(f+g) \prec \nu]=[f+g \prec-\nu]^{\perp}=[\bigvee[f \prec-\lambda] \wedge[g \prec-\mu]:-\lambda-\mu<-\nu]^{\perp}\right] \wedge[f \prec$ $-\lambda]^{\perp} \vee[g \prec-\mu]^{\perp}: \quad \lambda+\mu>\nu \geq \bigvee[f \prec-\lambda]^{\perp} \wedge[g \prec-\mu]^{\perp}: \lambda+\mu<\nu$ (since $\lambda^{\prime}+\mu^{\prime}>\lambda+\mu$ only if $\lambda^{\prime}>\lambda$ or $\left.\mu^{\prime}>\mu\right)=\bigvee[-f \prec \lambda] \wedge[-g \prec \mu]$ : $\lambda+\mu<\nu=[-f+-g \prec \nu]$. However, $-f$ is not quite an additive inverse, since $[f+-f \prec \nu]=\bigvee[f \prec \lambda] \wedge[f \prec \mu]^{\perp}: \lambda-\mu<\nu$ is $\varnothing$ for $\nu<0$ and $\bigvee[f \prec \lambda] \wedge[f \prec \mu]^{\perp}: \mu<\lambda=\bigvee[f \prec \lambda] \wedge \bigvee[f \prec \mu]^{\perp}$ for $\nu>0$ - to have the zero function, the last expression should be $X$. This coincidence may be taken as the definition of "finite-valued"; the finite-valued functions constitute a convex subset closed for lattice combinations, scalar multiplication, and sum. In general, $f+-f$ is a positive function, dominated by $|f|$ which absorbs it additively, hence is an "infinitesimal" with respect to $f$. Conversely, if $f$ is additively invertible and its positive multiples dominate some $g$, then for every $\lambda>0,[g \prec \lambda] \geq \bigvee[f \prec n \lambda]=X$, so $g \leq 0$.

In summary, on a Boolean $\sigma$-algebra, the distribution functions form a lattice-ordered additive monoid equipped with an order-inverting involutory negation which induces the additive inverse on the finite-valued ones-just those which have no proper infinitesimals - which constitute a convex (i.e. "solid") lattice- and scalar-closed group submonoid.

\section{DISTRIBUTION FUNCTION REPRESENTATION OF VECTOR LATTICES}

On a vector lattice, $(f-\lambda e)^{+}$is (for fixed positive $e$ ) a positive-valued form, antitone in $\lambda$, complete join-preserving and "reciprocally positive homogeneous" in $f$ : i.e. $(\pi f-\lambda e)^{+}=\pi\left(f-\pi^{-1} \lambda e\right)^{+}$for positive scalars $\pi$, and subadditive: i.e. $[(f+g)-(\lambda+\mu) e]^{+} \leq(f-\lambda e)^{+}+(g-\mu e)^{+}$. Thus the orthogonal complement, or annihilator, of any such form is, for fixed $f$, a distribution function on the complete Boolean algebra of annihilators; and this representation for the $f$ 's preserves all existent joins (complementation converting join to intersection), positive scalar multiplication, and is subadditive (the image of the sum is $\leq$ the sum of the images). ${ }^{2}$ 
These properties will assure that the representation preserves (possibly infinite existent) meets, negative scalar multiplication, and sum with vectors having finite-valued representatives, ${ }^{3}$ once it is seen that it preserves negation. The requirement for the representative of $-f$ to be the negative of that for $f$ reads: $(-f-\lambda e)^{+\perp}$, i.e. $(f+\lambda e)^{-\perp}, \perp(f+\kappa e)^{+\perp}$ for $\kappa>\lambda$ as well as $\geq(f+\mu e)^{+}$i.e. $(f+\lambda e)^{-} \perp(f+\mu e)^{+}$for $\mu<\lambda$. The latter holds because $(f+\lambda e)^{-} \perp(f+\lambda e)^{+} \geq(f+\mu e)^{+}$; the former cannot be expected in general since any nonzero element $\perp$ to both $f$ and $e$ will be in the intersection of these complements. However, if $e^{\perp}=0$-or better, by taking all complements relative to $e^{\perp \perp 4}$-one gets the desired disjointness of these elements' complements: when $\kappa>\lambda,(f+\kappa e)^{+} \geq(f+\lambda e)^{+}$and $(f+\lambda e)^{-} \geq(f+\kappa e)^{-}$; thus any element in both complements would be disjoint from both $f+\lambda e$ and $f+\kappa e$, hence from $e$.

Since the complements defining $[f \prec \lambda]$ are now being taken in $e^{\perp \perp}$, the vectors $f$ which, with their negative, are represented by 0 are just those for which $( \pm f+\lambda e)^{+\perp} \subset e^{\perp}$ for all $\lambda>0$-adding these to any vector will not change its representative. Every $f \in e^{\perp}$ as well as every $f$ infinitesimal with respect to $e$ satisfies these inclusions; conversely, the only vectors in $e^{\perp \perp}$ which are represented by negatives are those dominated by all positive $\lambda e$ : for $(f-\lambda e)^{+\perp} \supset e^{\perp \perp}$, i.e. $(f-\lambda e)^{+} \in e^{\perp}$, entails (since also $\left.\in e^{\perp \perp}\right) f \leq \lambda e$; thus if only zero is infinitesimal with respect to $e$, then only zero in $e^{\perp \perp}$ is represented by 0 for $\lambda<0, e^{\perp \perp}$ for $\lambda>0$. Under this condition also at most one vector in $e^{\perp \perp}$ is represented by any finite-valued distribution function: for if $f$ and $g$ had the same finite-valued image then $f-g$ would have zero image, hence be zero. Thus the representation, restricted to the part of $e^{\perp \perp}$ with finitevalued image, preserves the full group structure and is faithful when $e$ has no nonzero infinitesimal.

A vector in $e^{\perp \perp}$ is represented by a finite-valued distribution function just when there is no nonzero vector infinitesimal with respect to it: Indeed, if for a positive $f$ (recall that $f$ and $|f|$ are finite-valued together) $\bigvee[f \prec \lambda]$ had a proper complement in $e^{\perp \perp}$, then there would be an $e \geq g>0 \perp(f-\lambda e)^{+\perp}$ for all $\lambda>0, g \in(f-\lambda e)^{-\perp}=\left(e-\lambda^{-1} f\right)^{+\perp}$. Thus $\left(g-\lambda^{-1} f\right)^{+} \leq\left(e-\lambda^{-1} f\right)^{+} \in$ $g^{\perp}$; but also $\leq g^{+} \in g^{\perp \perp}$, whence $g \leq \lambda^{-1} f$ for all $\lambda>0$. The converse follows by the representation. Restricted to the solid vector sublattice of vectors without infinitesimals, the representation preserves additive inverse, thus the group, as well as the scalar and lattice, structure. In particular, an Archimedean space with unit is faithfully and densely represented, with preservation of its vector and complete lattice structure, in the space of distribution functions over the Boolean algebra of its annihilators.

One can obtain a global representation, faithful on the solid vector sublattice of vectors without infinitesimals, as follows. There is a natural notion of "restricting" a Boolean-valued distribution function to a principal ideal in the algebra, construing this as a Boolean algebra with the generator as unit and 
(more generally) complementation as relative to the generator: just compose the distribution function with meet with the generator. Now the distribution function representing $f$ in the ideal generated by $e^{\perp \perp}$ is the restriction of that in the ideal generated by the larger $\left(e^{\prime}+e\right)^{\perp \perp}$ when $e^{\prime} \perp e$ : for since $\lambda e^{\prime} \in e^{\perp}$, it is represented by 0 in the smaller ideal, whence $\left[f-\lambda e^{\prime} \prec \lambda\right]$ has the same value as $[f \prec \lambda]$ there. Thus values of $[f \prec \lambda]$ in the various $e^{\perp \perp}$ are coherent under restriction, for the partial order of disjoint summand on the $e$ 's; conversely the join, in the complete Boolean algebra of complements, of such coherent values at the finite sums of a pairwise orthogonal family of $e$ 's, yields the value of a distribution function at the Boolean ideal generated by their $e^{\perp \perp}$, whose restrictions have these values ${ }^{5}$-in particular, for a maximal orthogonal set, a distribution function on the whole algebra. If this set is chosen to extend a set maximal in the vectors without infinitesimals, then this solid vector sublattice is represented faithfully. Indeed, for a positive $f$ represented by a negative function, $(f-\lambda e)^{+} \in e^{\perp}$ for every (finite sum of) $e$ and $\lambda>0$; for the $e$ without infinitesimals $\bigvee(f-\lambda e)^{+}: \lambda>0=f \in e^{\perp}$ and so for $f$ without infinitesimals, i.e. $\perp \cap$ these $e^{\perp}, f=0$.

Since the distribution functions on a complete algebra form a complete lattice in which the finite-valued ones form a solid vector sublattice, this furnishes an alternate proof that every Archimedean vector lattice is embeddable in a boundedly (i.e. Dedekind) complete vector lattice. One can also derive the same representation for commutative lattice-ordered groups. Every such group is a subdirect product of totally-ordered groups (Lorenzen) and each of these is embeddable in a totally-ordered real vector space (Hahn)-thus every commutative lattice-ordered group is embeddable in a vector lattice. The rational vector subspace generated by the embedded group is canonically determined (as is the vector sublattice generated by the $l$-subgroup of elements without proper infinitesimals). No group element without proper infinitesimals would obtain one in the generated rational vector space; nor-since the latter is cointial in its positives-in the generated vector lattice. Thus one need only apply the above representation to this vector lattice to obtain the corresponding representation for the embedded l-group.

\section{DISTRIBUTION FUNCTIONS AS CONTINUOUS OR MEASURABLE FUNCTIONS}

The proof that the system of distribution functions on a Boolean $\sigma$-algebra is just the system of extended real-valued continuous functions on its Stone space will not be reproduced here (see [F]). It should only be mentioned that since a nonzero distribution function is nonzero on some clopen subset of the Stone space, the "finite-valued" distribution functions are only guaranteed not to be infinite on any clopen, i.e. they are finite-valued on a dense subset of the Stone space. Conversely, since any densely finite-valued point function does not dominate all scalar multiples of any continuous function, its distribution function is "finite-valued." 
Recall that a Boolean algebra is $\sigma$-complete if and only if in its Stone space the closure of every open $F_{\sigma}$ is (cl)open; it is complete if and only if the closure of every open set in its dual is (cl)open-these latter spaces are called "extremally disconnected". Thus the distribution functions on a complete algebra are just the extended real-valued continuous functions on its extremally disconnected Stone space, the "finite-valued" ones being just those which are densely finite-valued. Combining with the above representation in the distribution functions, one obtains a representation of vector lattices as dense sublattices of continuous functions on extremally disconnected spaces, the vectors without infinitesimals being represented faithfully and with preservation also of their algebraic operations in the densely finite-valued functions. In particular, an Archimedean vector lattice is faithfully and algebraically represented as a dense vector sublattice of densely finite-valued continuous functions. This last is the main result in the subject; it has been proved here for $l$-groups as well.

Alternatively, the system of distribution functions on a Boolean $\sigma$-algebra may be identified with the equivalence classes of measurable functions by appealing to the Loomis-Sikorski representation of the $\sigma$-algebra as a quotient of a $\sigma$-field modulo a $\sigma$-ideal of subsets of some set. ${ }^{6}$ In general, every distribution function on an algebra passes back to any other algebra of which the given one is a homomorphic image: if $[f \prec \lambda]$ has been lifted for a finite number of $\lambda$ 's then one for a further $\lambda$ can be lifted so as to preserve isotoneness by meeting any of its inverse images with the smallest lifted $[f \prec \mu]$ for $\mu>\lambda$ and joining with the largest for $\mu<\lambda$ : thus $[f \prec \lambda]$ can be lifted isotonely for all rational $\lambda$. Two such liftings differ by a map of the (unlifted) $[f \prec \lambda]$ into the kernel; every distribution function on the domain is such a lift of its composition with the quotient morphism.

Every distribution function to a field of subsets is equivalent to the inverse image of $(-\infty, \lambda)$ for a unique real-valued function on the support of the field. This function must obviously take the value $f x=\bigwedge \lambda: x \in[f \prec \lambda]$ (since $f x=\mu$ iff $\lambda>\mu \rightarrow x \in[f \prec \lambda]$ and $\lambda<\mu \rightarrow x \notin[f \prec \lambda])$. Thus it is measurable with respect to the generated $\sigma$-field; conversely, every such measurable function has as inverse image a distribution function to this $\sigma$-field.

Consider now a $\sigma$-field $\Sigma$, of subsets of some set $X$, containing a $\sigma$-ideal $\mathscr{N}$. Every distribution function with values in the quotient $\sigma$-algebra $\Sigma / \mathscr{N}$ lifts to $\Sigma$ uniquely modulo $\mathscr{N}$ and so determines a measurable $(\Sigma)$ real-valued function on $X$ unique up to equivalence $\bmod \mathscr{N}$; conversely, the distribution function of every measurable function composed with the quotient map mod $\mathscr{N}$ yields a $\Sigma / \mathscr{N}$-valued distribution function, the same one being obtained for just the $\mathscr{N}$-equivalent measurable functions. There is thus a one-to-one correspondence between the classes of $\mathscr{N}$-equivalent measurable functions and the distribution functions on $\Sigma / \mathscr{N}$. This correspondence is, moreover, an order-isomorphism, whence it preserves all existent lattice extrema on either side; when $\Sigma / \mathscr{N}$ is complete (as it will be in a moment) these will both be 
complete lattices. A class of measurable functions is "finite-valued"-has no nonzero infinitesimal-just when it consists of a.e. finite functions.

It has been shown that every Archimedean vector lattice is completely embeddable in the finite-valued distribution functions on the complete Boolean algebra of its annihilators. By the Loomis-Sikorski theorem this algebra can be represented as the quotient of a $\sigma$-field $\Sigma$ on some set $X$ modulo a $\sigma$-ideal $\mathcal{N}$. Thus by the above, the complete vector lattice embedding can be realized in the classes $\bmod \mathscr{N}$ of a.e. finite measurable functions on $X$.

The spectral representation in the vector lattice of measurable functions is a simple translation of measurability. To every finite-valued measurable function $f$ assign the "spectral system" $e_{\lambda}=$ characteristic function of (its distribution) $f^{-1}(-\infty, \lambda)$-this satisfies the list of [V, p. 103, Lemma IV.10.1]. For every countable partition of the reals into left closed right open intervals and every choice of $\rho \in[\mu, \lambda)$, the sum $\Sigma \rho\left(e_{\lambda}-e_{\mu}\right)$ is a finite-valued measurable function; these converge uniformly a.e. (thus relatively uniformly with respect to the constant 1 as regulator) to $f$ as $\sup (\lambda-\mu)$ goes to zero, which is written

$$
f=\int_{-\infty}^{\infty} \lambda d e_{\lambda} .
$$

It is usual to require the spectral system $e_{\lambda}$ as well as the approximating sums to belong to the original vector lattice rather than to the containing lattice of measurable functions in which it is embedded. Now if $e$ is the characteristic function of a measurable set, $e_{\lambda} e=e_{\lambda} \wedge e$ may be obtained vector lattice theoretically as $\bigvee_{n} n(\lambda e-f)^{+} \wedge e$ and so it would suffice to require these sups to exist for some maximal pairwise orthogonal family of $e$ 's in the original vector lattice which also includes the $f e$ (these may be obtained intrinsically when the projections of $f$ on $e^{\perp \perp}$ exist). Then the truncations $\lambda e \wedge f e \vee \mu e$ of each $f e$ are spectral representable as above using only (the available) finite approximating sums and since $\lambda e \wedge f e \vee \mu e$ order converges to $f$ as $\lambda \uparrow \infty$, $\mu \downarrow-\infty, e \uparrow 1$ (indeed, positive and negative parts separately $\uparrow$ to those of $f$ ) $f$ will be spectral representable if its integral is defined as the order limit of the uniformly convergent integrals of its truncated restrictions to the $e$. The requisite sups exist if the lattice is boundedly ("Dedekind") $\sigma$-complete (since the sups are bounded by $e$ ) or, more generally, if it has the "principal projection property" which is the existence of $\bigvee_{n} n f \wedge g$ for all $f, g \geq 0$.

The representation can also be used to derive ${ }^{7}$ Kakutani's representation of "L-spaces". Recall that a "lattice norm" \|\| on a vector lattice is a vector space norm for which $\|f\| \leq\|g\|$ whenever $|f| \leq|g|$ (thus \|\| is factored by || ); if the space is complete for this norm it is called a Banach lattice. This is called an (abstract) $L$-space when the norm satisfies $\|f+g\|=\|f\|+\|g\|$ for $f, g \geq 0$.

In such a space an order-bounded updirected net has a supremum to which it norm converges [KN, 24.10]. In any Banach lattice the norm sends a positive updirected order-bounded $f_{n}$ to a similar real $\left\|f_{n}\right\|$ which thus converges; in an $L$-space $\left\|f_{n}-f_{m}\right\|=\left\|f_{n}\right\|-\left\|f_{m}\right\|$ for $n \geq m$, hence the $f_{m}$ are norm (Cauchy, 
hence) convergent and the limit $f$ is still $\geq f_{m} \quad\left(\left|f_{m} \wedge g-f_{m} \wedge h\right| \leq|g-h|\right.$ shows $f_{m} \wedge$ norm continuous); no $f^{\prime}<f$ could bound the $f_{m}$ since $\left\|f-f_{m}\right\|=$ $\left\|f-f^{\prime}\right\|+\left\|f^{\prime}-f_{m}\right\| \geq\left\|f-f^{\prime}\right\|$ would contradict convergence. An $L$-space is thus boundedly complete as a vector lattice and so is represented by a vector sublattice of measurable functions which includes the values of their distribution functions. Then one can find, for each representing positive measurable function, an increasing sequence of simple functions in this vector sublattice, of which it is the a.e. sup. On the characteristic functions the norm induces an ordinary non-negative real-valued measure and on the simple functions it agrees with the integral of the absolute value-thus this sequence of simple functions is mean Cauchy and a.e. convergent to the measurable function which is thus (by definition) integrable for this measure with value of its integral equal to its norm.

\section{ENDNOTES}

${ }^{1}$ This gives the pointwise sum where either factor is finite: $f$ takes on the value $+\infty$ on the complement of $\bigvee[f \prec \lambda]$ and the sum will be $+\infty$ there as well; it is $-\infty$ on $\Lambda[f \prec \lambda]$ which will be the value of the sum insofar as $g \neq+\infty$. Adjoining an absorbing element to an associative commutative system does not destroy these properties; what one has done here is to adjoin $-\infty$ as an absorbing element to the finite reals and then adjoin $+\infty$ as absorbing element to the augmented system.

2 It also preserves finite meets since $f \rightarrow f^{\perp}$ converts finite meets of positive elements to Boolean joins (using that $f \perp S$ entails $f^{\perp \perp} \perp S$, pass from $f \wedge g \wedge(f \wedge g)^{\perp}=0$ to $f^{\perp \perp} \wedge g^{\perp \perp} \wedge$ $\left.(f \wedge g)^{\perp}=0\right)$.

${ }^{3}$ Or even only whose self-difference is absorbed on being added to the representative of the sum-e.g. all sums of positives will be preserved.

${ }^{4}$ Since $e$ is now sent on the constant function 1 , this makes the image of the vector lattice dense (have $0 \perp$ ) in the distribution functions.

${ }^{5}$ Since only the zero distribution function is $\perp$ to the images of all the $e$ 's, the image of the vector lattice is again dense.

${ }^{6}$ See $[S, \S \S 29,32,43]$, where this is done with a somewhat different notion of distribution function and references to other treatments may be found.

${ }^{7}$ A similar derivation, less effective since it uses the continuous function representation, can be found in $[A B$, p. 192f].

\section{REFERENCES}

[AB] C. Aliprantis and O. Burkinshaw, Positive operators, Academic Press, New York, 1985.

[AF] M. Anderson and T. Feil, Lattice-ordered groups, Reidel, Dordrecht, 1988.

[B] G. Birkhoff, Lattice theory, Amer. Math. Soc. Colloq. Publ. 25 (1967).

[BKW] A. Bigard, K. Keimel and S. Wolfenstein, Groupes et Anneaux Réticulés, Lecture Notes in Math. No. 608, Springer, Berlin, 1977.

[F] I. Fleischer, "Place functions": alias continuous functions on the Stone space, Proc. Amer. Math. Soc. 106 (1989), 451-453.

[H] P. R. Halmos, Measure theory, Van Nostrand, New York, 1950.

$\left[\mathrm{H}^{\prime}\right]$ _ Lectures on Boolean algebras, Van Nostrand, Reinhold, New York, 1963.

[KN] J. L Kelley, I. Namioka et al., Linear topological spaces, Princeton, 1963. 
[LZ] W. A. J. Luxemburg and A. C. Zaanen, Riesz spaces I, North-Holland, Amsterdam, 1971.

[S] R. Sikorski, Boolean algebras, Springer, Berlin, 1960.

[V] B. Z. Vulikh, Introduction to the theory of partially ordered spaces, Walters-Noordhoff, Groningen, 1967.

Department of Mathematics, University of Windsor, Windsor, Ontario N9B 3P4 CANADA 